\title{
Image-based Orientation Determination of Mobile Sensor Platforms
}

\author{
O. Hasler *, S. Nebiker \\ Institute of Geomatics Engineering, FHNW University of Applied Sciences and Arts Northwestern Switzerland, \\ Muttenz, Switzerland - (oliver.hasler, stephan.nebiker)@fhnw.ch
}

KEY WORDS: Orientation estimation, image-based, panorama images, robotics, mobile sensor platforms

\begin{abstract}
:
Estimating the pose of a mobile robotic platform is a challenging task, especially when the pose needs to be estimated in a global or local reference frame and when the estimation has to be performed while the platform is moving. While the position of a platform can be measured directly via modern tachymetry or with the help of a global positioning service GNSS, the absolute platform orientation is harder to derive. Most often, only the relative orientation is estimated with the help of a sensor mounted on the robotic platform such as an IMU, with one or multiple cameras, with a laser scanner or with a combination of any of those. Then, a sensor fusion of the relative orientation and the absolute position is performed. In this work, an additional approach is presented: first, an image-based relative pose estimation with frames from a panoramic camera using a state-of-the-art visual odometry implementation is performed. Secondly, the position of the platform in a reference system is estimated using motorized tachymetry. Lastly, the absolute orientation is calculated using a visual marker, which is placed in the space, where the robotic platform is moving. The marker can be detected in the camera frame and since the position of this marker is known in the reference system, the absolute pose can be estimated. To improve the absolute pose estimation, a sensor fusion is conducted. Results with a Lego model train as a mobile platform show, that the trajectory of the absolute pose calculated independently with four different markers have a deviation $<0.66$ degrees $50 \%$ of the time and that the average difference is $<1.17$ degrees. The implementation is based on the popular Robotic Operating System ROS.
\end{abstract}

\section{INTRODUCTION}

The precise estimation of position and orientation of robots or autonomous vehicles is becoming increasingly important. The challenge is the highly precise estimation of these values in a kinematic system. Position can be determined in near real-time using various approaches such as motorized tachymeters indoors and outdoors or GNSS outdoors. However, determining the exact orientation or orientation of these platforms is challenging. Conventional Visual SLAM/Visual Odometry approaches can determine position and orientation, but only relative to their initial position. In an unpublished research project of the Institute of Geomatics (IGEO) at the FHNW, the orientation of a platform is calculated with high accuracy using a motion capture system carried on board the mobile platform. However, the setup of this system is complex and scalable only to a limited extent.

In this work, an alternative image-based approach to determine the orientation in three Degrees of Freedom (DoF) using panoramic cameras was developed. Panoramic cameras have the advantage of capturing the entire environment of a platform and provide a more geometrically robust orientation determination than classical cameras. In addition, this work developed and investigated methods to determine the absolute orientation of a mobile sensor platform with respect to a reference frame.

\section{RELATED WORK}

Image-based orientation estimation is a widely researched topic and several differentiations between its methods can be made. Feature-based methods try to extract image features (such as parallel lines, vanishing points or SIFT Features) and track these features over different frames. This method needs additional image processing steps while extracting and tracking these features and is sensitive to outliers and illumination change. On the other hand, appearance-based methods directly compare pixel intensities of the overall image. This approach is also referred to as dense, direct or global, depending on the context (Irani and Anandan, 2000).

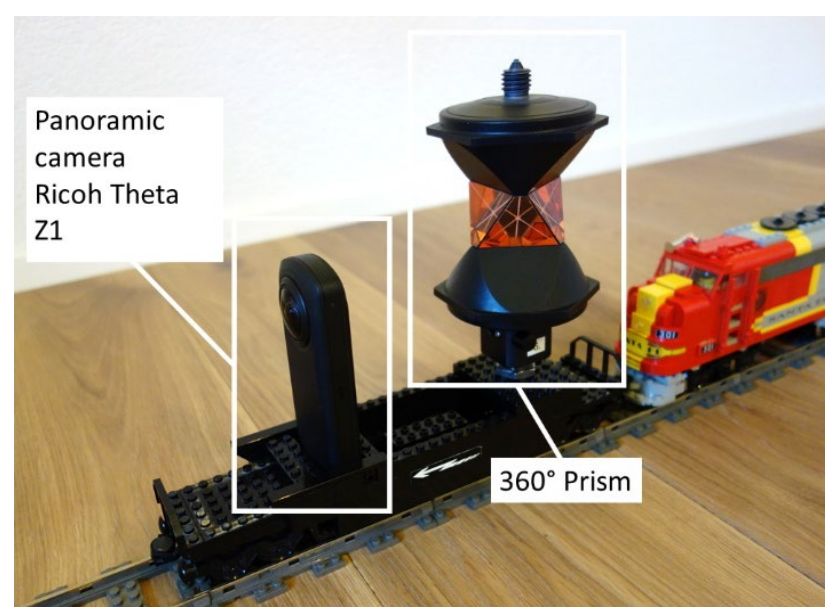

Figure 1. Mobile sensor platform with panoramic camera Ricoh Theta $\mathrm{Z} 1$ and $360^{\circ}$ prism mounted on a LEGO model train

Another criterion for distinguishing image-based orientation estimation is the estimated degrees of freedom (DoF). Visual compass methods limit themselves to a single axis (yaw). In the Visual Compass approach of Morbidi and Caron (2017), only the horizontal direction change is determined from the phase correlation of two consecutive images. Visual gyroscopes estimate the attitude (roll, pitch and yaw angles). The two approaches of Hartmann et al. (2015) are either real-time capable but too inaccurate or not real-time capable. Caron and Morbidi (2018) extended their Visual Compass approach to 
Visual Gyroscope, but with a computation time of 1 second per image this approach is also not operational for our application. Finally, Visual Odometry approaches are used to determine the camera pose (orientation and position). The indirect approach of Mur-Artal and Tardos (2017) is state-of-the-art, but panoramic cameras cannot be used. Sumikura et al. (2019) have extended this approach so that $360^{\circ}$ cameras can also be used. However, all approaches work in a relative coordinate system or can relocate in an already acquired map.

Image-based absolute orientation determination of a mobile platform is an area that has not been extensively explored. Approaches from Visual Localization, in which an image is oriented with respect to reference images, are described in Sattler et al. (2018). However, this requires a reference model consisting of already oriented images. Another possibility would be to calculate the orientation from the trajectory of an absolute positioning method (e.g. tachymetry). However, only the azimuth and the inclination in the direction of travel can be determined, not the lateral inclination.

\section{MOBILE PLATFORM AND TEST SITE}

This work aims at image-based orientation determination for a variety of mobile platforms: mobile robots or the tip of a robotic arm, vehicle monitoring or mobile measurement platforms. For feasibility reasons (including lab accessibility restrictions caused by the Covid19 pandemic), a highly dynamic Lego train was used as a mobile sensor platform for this work at the home office. This platform has the advantage of ensuring repeatability of manoeuvres. In addition, the sensors can be fixed and easily arranged. A Ricoh Theta Z1 panoramic camera and a $360^{\circ}$ prism are mounted on the platform (Figure 1). A Leica MS60 totalstation and a coded ArUco (Garrido-Jurado et al., 2014) marker with a size of $19 \times 19 \mathrm{~cm}$ are installed in the room (Figure 2).

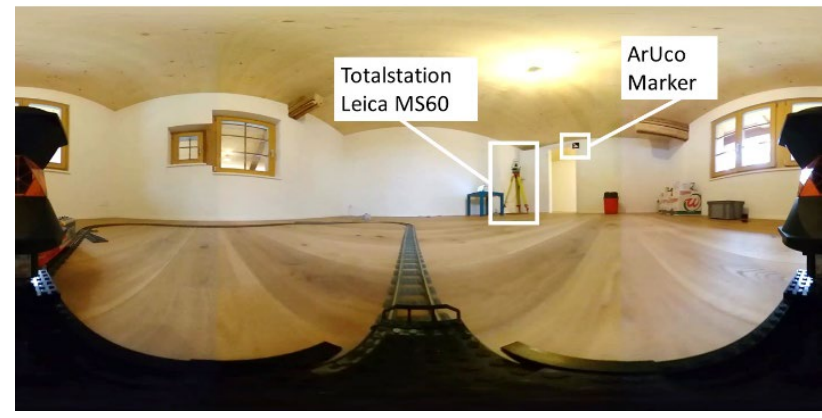

Figure 2. Equirectangular camera image depicting totalstation and marker (right) together with $360^{\circ}$ prism (at the left and right image border)

By kinematically tracking the $360^{\circ}$ prism with the totalstation Leica MS60, the position of the platform is determined with high precision at a clock rate of $19 \mathrm{~Hz}$. The orientation calculation is performed with the Ricoh Theta Z1 panoramic camera (30 FPS, video resolution $3840 \times 1920$ pixels). The panoramic camera is factory calibrated and a built-in method allows to convert the sensor images into a single equirectangular panoramic image. Unfortunately, it is currently impossible to transmit the video stream of the camera in (near) real-time, therefore, the video stream was recorded, and all further calculations were made in post processing.

\section{IMAGE-BASED ORIENTATION ESTIMATION}

Orientation determination can be divided into three parts: Relative orientation estimation, position determination and absolute orientation estimation (Figure 3). The relative orientation takes place in the local coordinate system odom frame, the position determination and the absolute orientation estimation in the global system map frame. Finally, a sensor fusion is applied to obtain an absolute pose in the map frame.

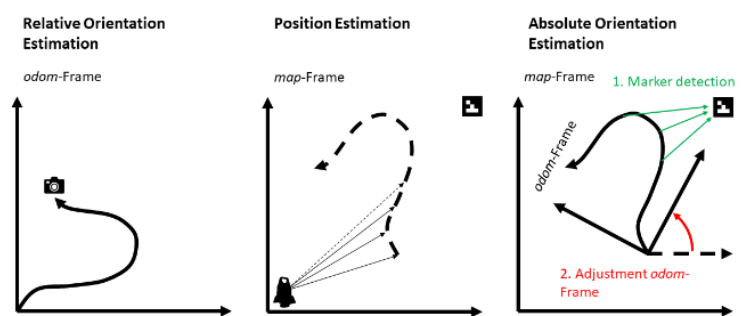

Figure 3. Concept of the three parts of the absolute orientation estimation: relative orientation (left), position (centre) and absolute orientation (right) with the respective reference frames

\subsection{Relative Orientation Estimation}

The relative orientation calculation is performed using OpenVSLAM (Sumikura et al., 2019). OpenVSLAM was successfully implemented in the program flow and optimized for real-time computation. OpenVSLAM is ideally suited because this implementation supports different camera systems (monocular, stereo, RGBD) and different camera models (perspective, fisheye, dual fisheye, catadioptric) with different imaging models (including equirectangular). In addition, OpenVSLAM already provides a ROS implementation that computes the relative orientation from a ROS camera stream. The trajectory with the orientations refers to the odom frame coordinate system with the coordinate origin at the initialization location.

\subsection{Position Determination}

The position of the mobile platform is continuously determined using a Leica MS60 multistation. For this purpose, an ROS node with time synchronization was developed at the Institute of Geomatics, which can control the MS60 and receive measured values. The multistation is stationed in space with respect to a defined coordinate system (map frame). Subsequently, the multistation continuously tracks the $360^{\circ}$ prism, which is mounted on the mobile platform. The measurement rate is about $19 \mathrm{~Hz}$ and the expected 3D accuracy is about $5 \mathrm{~mm}$. As soon as the platform moves, a rough horizontal orientation (heading/yaw) can be calculated from the trajectory. This is calculated from the two positions at time $t$ and $t-1$ and used as a rough control for the absolute orientation calculation.

\subsection{Absolute Orientation Estimation}

The main part of this work is the determination of the absolute orientation. This determination is necessary because a) the orientation determination by means of OpenVSLAM only yields the relative orientation with respect to the initialization orientation and b) the orientation just from position determination provides an insufficiently accurate orientation. To calculate the absolute orientation, at least one ArUco marker must be attached in space. The position of the marker is measured with the multistation. Since the relative orientation may exhibit drift, the absolute orientation estimation is executed 
continuously. The absolute orientation estimation is performed as follows:

4.3.1 Initialisation Phase: With the first measurement, the translation of the odom frame into the map frame can be calculated. The origin of the odom frame is set to the prism position. The estimation of the translation needs to occur while the platform is not moving.

4.3.2 Check for recent Position: For every new camera frame, the time difference between the camera frame and the latest position estimation is estimated. If the difference is less than one microsecond, the camera frame will be used for further computation.

4.3.3 Marker Detection: In the case of a time-synced camera frame and prism position, an ArUco marker detection in the current camera frame is performed. For the detection, the OpenCV function cv2.aruco.detectMarkers (OpenCV: Detection of ArUco Markers, n.d.) with subpixel refinement is used. If a marker gets detected, the marker ID and the image coordinates of the marker corners are returned.

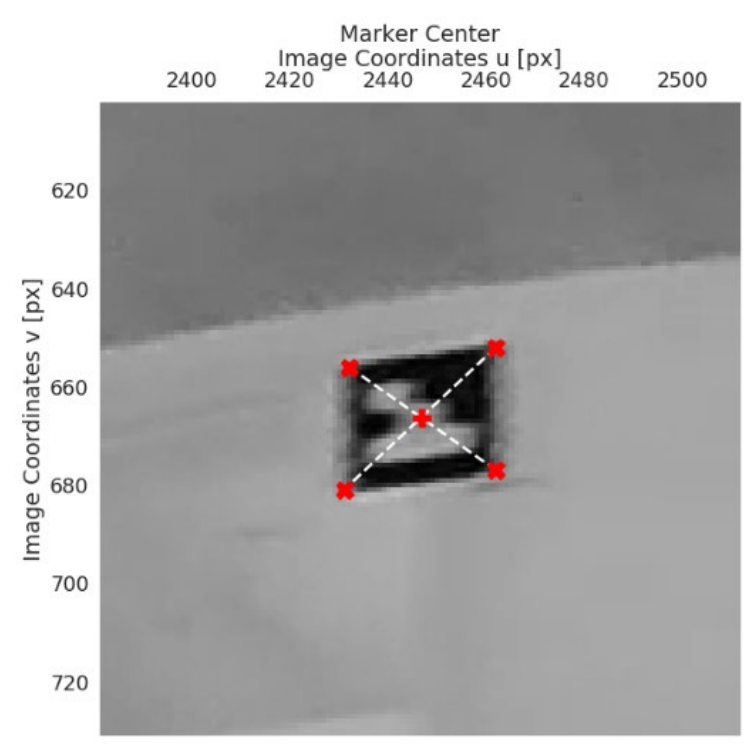

Figure 4. Detected marker in cropped camera frame

4.3.4 Marker Centre Calculation: Since the detected marker can be distorted, the marker centre is calculated by the intersection of the two diagonals of the marker corners (Figure 4).

4.3.5 Direction Calculation: From the coordinates of the marker centre and the image size, the horizontal and vertical direction of the marker in respect to the camera can be calculated. If the camera model is equirectangular, this calculation becomes simple, with other camera models or when using an multi head system, the intrinsic and relative camera calibration are needed (Figure 5).

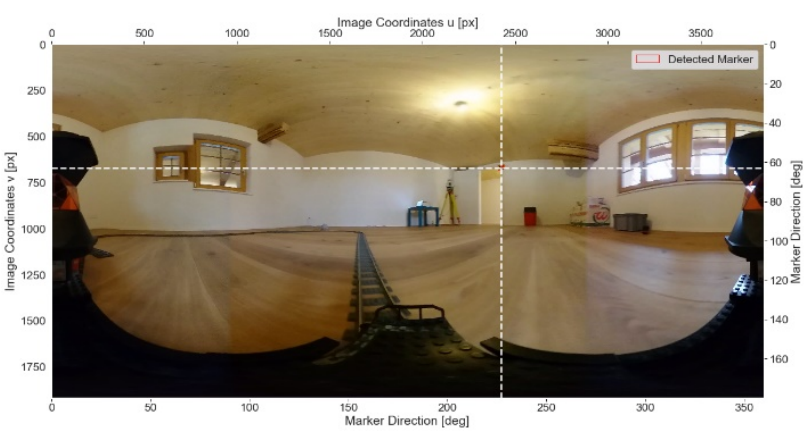

Figure 5. Horizontal and vertical direction of detected marker used for direction calculation in equirectangular camera frame

4.3.6 Calculation of possible Camera Locations: The possible camera locations can be calculated using the horizontal direction of the marker, the horizontal distance between $360^{\circ}$ prism and camera and the position of marker and $360^{\circ}$ prism (Figure 6).

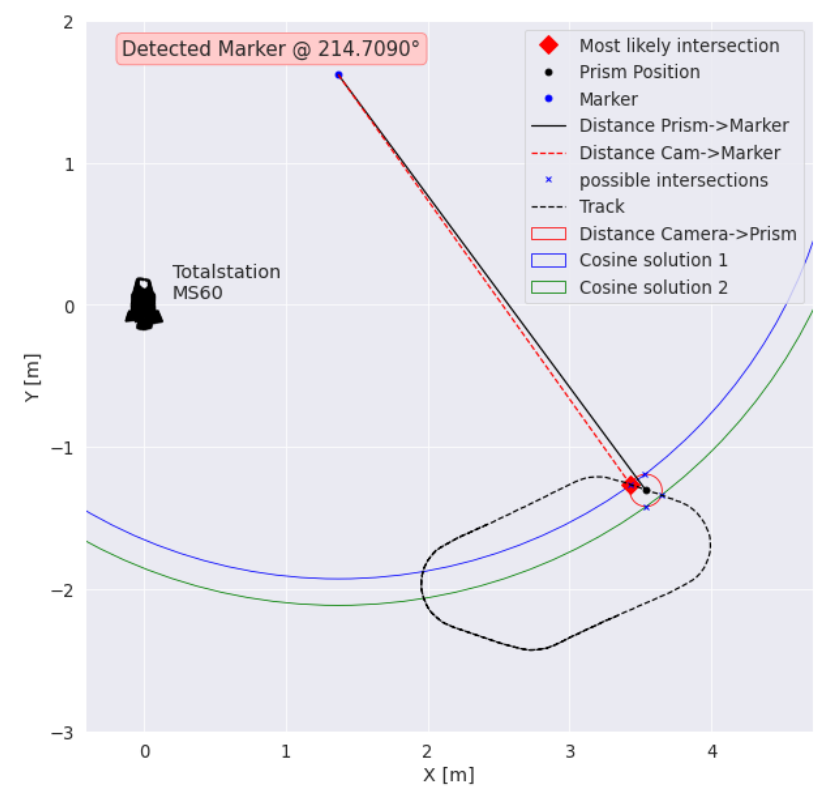

Figure 6. Calculation of possible camera locations: Current prism 3D position (black dot, position via tachymetry), marker 3D position (blue dot), cosine solutions (blue, green) intersected with leverarm camera->prism (red) gives possible camera positions (black crosses). Most likely position (red) in direction of travel.

The possible camera locations are calculated with the cosine theorem, which returns four possible locations. The location, which is in the direction calculated from the prism tracking is the most likely position and will be used.

4.3.7 Odom Frame Adjustment: Finally, the orientation of the odom frame gets adjusted and the odometry trajectory is absolutely oriented. During the initialization phase, the corrections are big, afterwards the corrections are only incremental and compensate only the drift of the odometry trajectory from the relative orientation estimation. 


\subsection{Sensor Fusion}

To obtain the best possible estimate of the mobile platform's pose and to take advantage of redundancies in the determination of position and orientation, the position determinations by the multistation and the absolute orientation are fused together. Therefore, absolute orientation and position can be published at the same rate as relative orientation determination. In addition, other sensors such as odometers (wheel odometry) or IMU could be included in the sensor fusion if they were available.

The sensor fusion is performed using the ROS package robot_localization (Moore and Stouch, 2016). This package can be used to configure a sensor fusion. The sensor fusion calculates the robot pose at $30 \mathrm{~Hz}$ (same rate as camera frames).

\section{EXPERIMENTS AND RESULTS}

\subsection{Sensor Synchronisation}

Due to the camera stream limitation, it was not possible to acquire a synced dataset of camera stream and prism tracking. Therefore, a sensor synchronisation needs to be conducted before the postprocessing. For the synchronisation, only the time offset between the two datasets needs to be found. The time offset can be determined via cross correlation of a common phenomenon, in this case it is the heading of the mobile platform. The heading from the relative orientation is compared with the heading calculated from the prism tracking. The used heading is the average between the heading towards the next prism position and the previous position. Since the frequency of the prism tracking is smaller than the frequency of the relative orientation, the calculated prism headings are interpolated to the same frequency (Figure 7).

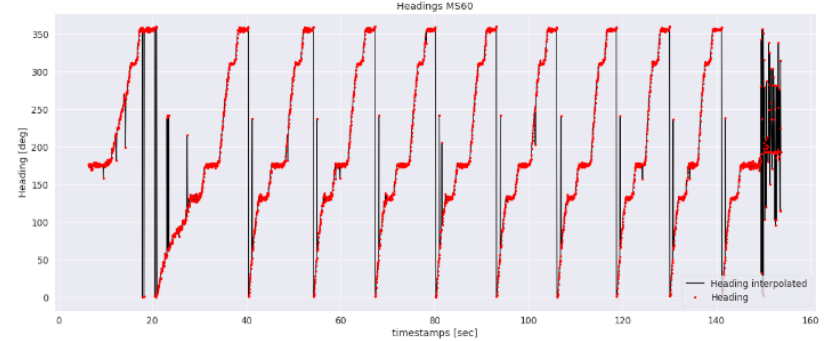

Figure 7. Interpolation of the prism headings: original (red) and interpolated (black)

Subsequently, the time offset between the measurements can be calculated. The biggest correlation for the dataset used in the evaluation (see Figure 8) occurred at an offset of 11 frames ( 0.367 seconds). This offset was used for further processing.

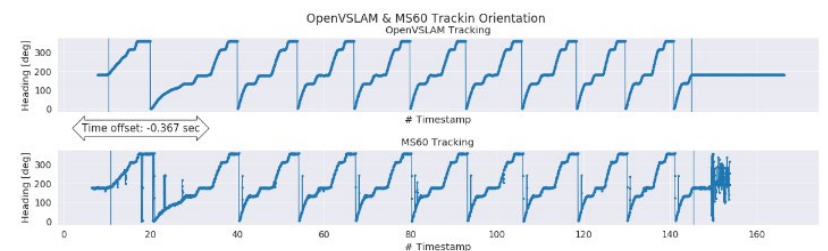

Figure 8. Time offset between relative orientation estimation (top) and prism tracking (bottom)

\subsection{Relative Orientation Estimation}

The relative orientation determination can be performed with different parameterizations. To be able to perform the determination in real-time, the resolution of the camera stream must be reduced, the number of features to be detected must be reduced and the loop closure detection must be deactivated.
However, the most continuous trajectory is obtained with the full resolution $(3840 \times 1920$ pixels) and loop closure detection disabled (Figure 9). With this configuration, the images can be computed at just under $8 \mathrm{~Hz}$. Since this configuration is not real-time capable, the relative orientation was calculated in advance for the further investigations.

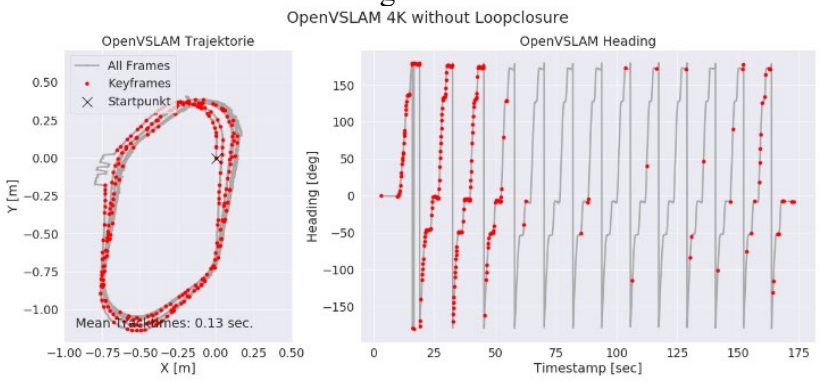

Figure 9. Horizontal drift: OpenVSLAM trajectory without loop closure for 13 laps (left) and evolvement of the horizontal drift (right)

\subsection{Marker Detection}

In the range study for the present combination of camera and ArUco markers, a marker was fixed in a large room and then a picture was taken with a panoramic camera at different distances from the marker. The marker could be successfully detected at a distance between 3 and 11 meters. From a distance of 12 meters, the marker could no longer be detected in the camera image. The detected marker still has a size of about $9 \times 9$ pixels in the image at a distance of 11 meters with the panorama camera used (Ricoh Theta Z1).

\subsection{Evaluation of the complete System}

To determine the accuracy of the absolute orientation, images were taken with the Lego Train platform in a room where four different markers (M1, M2, M3, M4) were placed at different locations (Figure 10).

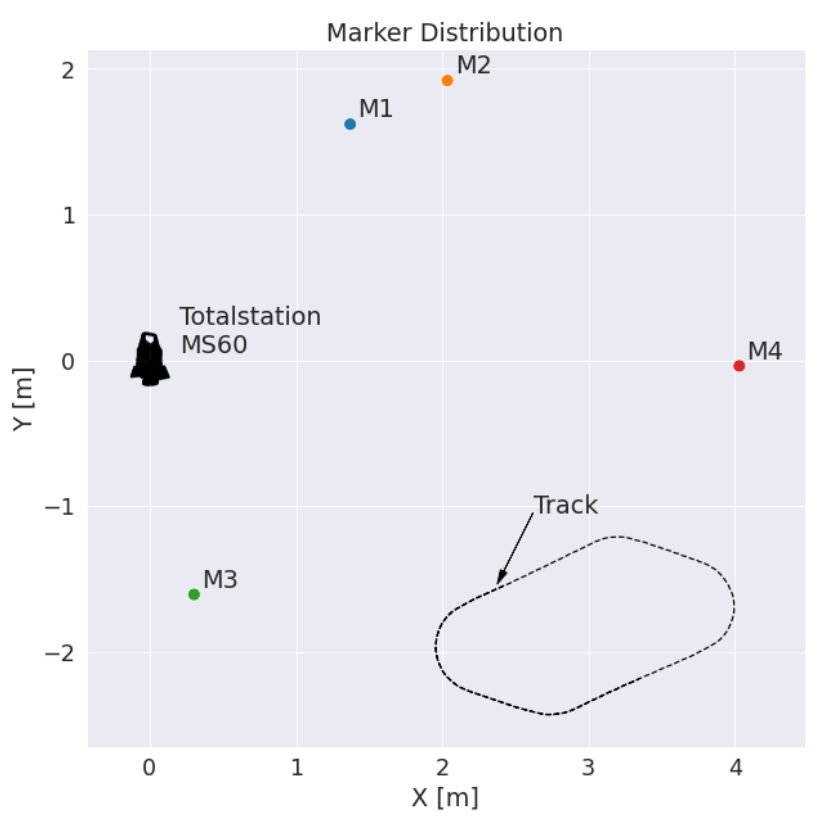

Figure 10. Marker distribution (M1 - M4) on test site for evaluation

Subsequently, the absolute orientations were calculated four times from the recordings. Each time, one of the four markers 
was used for the absolute orientation correction. In this process, the calculated poses from the sensor fusion were recorded and subsequently compared with each other.

The RPE (relative pose error, relative pose deviation) (Sturm et al., 2012) was used as a method for comparing every pose of the trajectory calculated using the four different markers. The RPE calculates the difference between two trajectories and is commonly used to compare an estimated/calculated pose with the true pose. The true pose is usually determined by another, more accurate method, such as a motion capture system. Since no such motion capture system was available for processing this work, only the trajectories calculated with different markers were compared. On average, the orientation deviation is 1.17 degrees with a mean standard deviation of 1.66 degrees.

Figure 11 shows the change in horizontal orientation of the calculated trajectories. On closer inspection, it is noticeable that the orientation deviates constantly depending on the marker used.

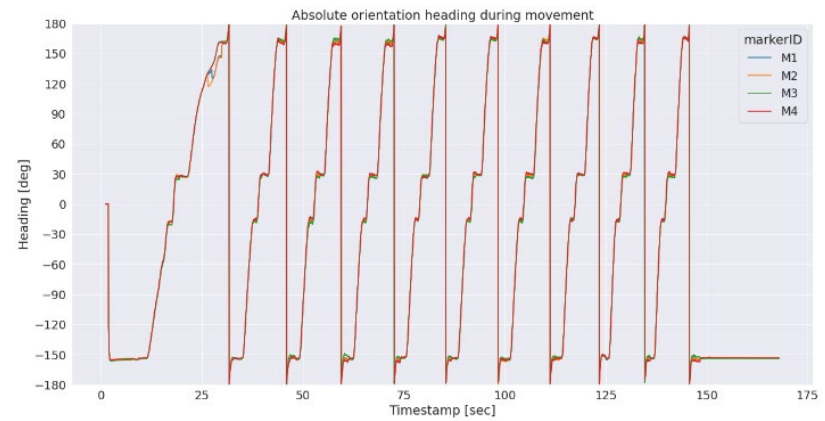

Figure 11. Heading of absolute orientation during 10 laps

Figure 12 shows the individual orientation adjustments with the respective marker used. There, too, at the beginning and the end (standstill of the platform) it is clearly visible that the that the adjustments show a significant bias. Since the deviations are constant and also occur at standstill, the assumption is obvious that the camera calibration still shows systematic errors.

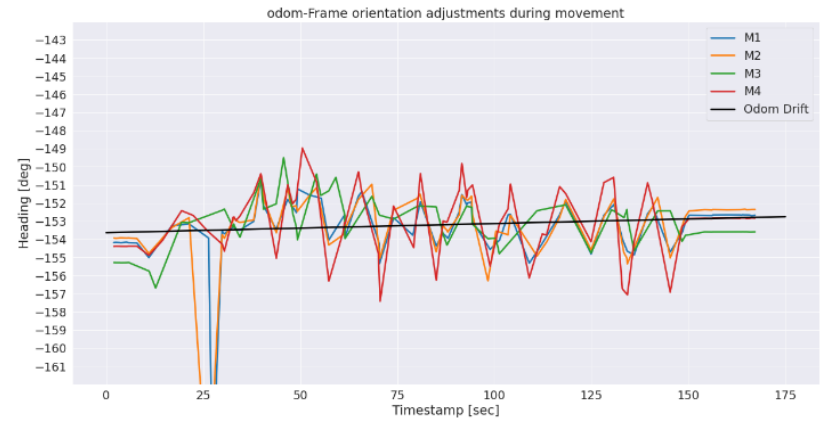

Figure 12. Adjustments on odom frame horizontal orientation over 10 laps

Further investigation into systematic errors were performed. A comparison between the place of the marker detection in the camera frame and the resulting rotation of the odom frame shows, that depending on where the marker gets detected in the camera frame, the odom frame gets adjusted differently (Figure $13)$.

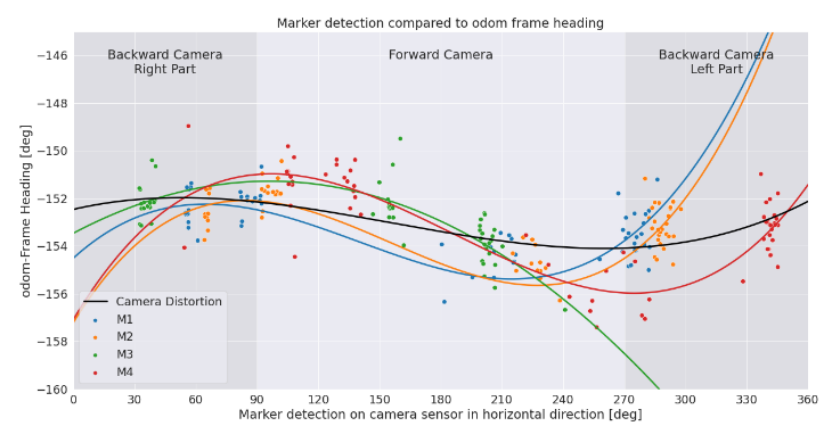

Figure 13. Comparison of position of the marker detection on the camera sensor to odom frame adjustment

\section{CONCLUSION AND OUTLOOK}

In this paper a new approach for high precision determination of absolute orientation was presented. The absolute orientation with an ArUco marker works well and it could be shown in a study with the Lego train platform that $50 \%$ of the determinations are more accurate than 0.66 degrees and the difference of the poses between the processed trajectories with different marker detection are on average 1.17 degrees. With this approach, the installation effort is very low: set up the total station, measure the markers, and the absolute orientation of the mobile platform can be determined. In order to increase the system accuracy, instead of using the factory calibration of the camera, a custom calibration would need to be performed and applied. Unfortunately, this was not possible in this work in the home office due to the special situation at the time of the investigations. In the future, multiple markers could be used to determine absolute orientation, thus all 6 degrees of freedom could be determined continuously. In addition, alternative markers could be searched for, which would increase the radius of action of the mobile platform.

\section{REFERENCES}

Caron, G., Morbidi, F., 2018: Spherical Visual Gyroscope for Autonomous Robots Using the Mixture of Photometric Potentials. Proceedings - IEEE International Conference on Robotics and Automation, 820-827.

https://doi.org/10.1109/ICRA.2018.8460761

Garrido-Jurado, S., Muñoz-Salinas, R., Madrid-Cuevas, F. J., Marín-Jiménez, M. J., 2014: Automatic generation and detection of highly reliable fiducial markers under occlusion. Pattern Recognition, 47(6), 2280-2292.

https://doi.org/10.1016/j.patcog.2014.01.005

Hartmann, W., Havlena, M., Schindler, K., 2015: Visual gyroscope for accurate orientation estimation. Proceedings 2015 IEEE Winter Conference on Applications of Computer Vision, WACV 2015, 286-293.

https://doi.org/10.1109/WACV.2015.45

Irani, M., Anandan, P.; 2000: About direct methods. Lecture Notes in Computer Science (Including Subseries Lecture Notes in Artificial Intelligence and Lecture Notes in Bioinformatics), 1883, 267-277. https://doi.org/10.1007/3-540-44480-7_18

Moore, T., Stouch, D.; 2016: A generalized extended Kalman filter implementation for the robot operating system. Advances in Intelligent Systems and Computing, 302, 335-348. https://doi.org/10.1007/978-3-319-08338-4_25

Morbidi, F., Caron, G., 2017: Phase Correlation for Dense 
Visual Compass from Omnidirectional Camera-Robot Images. IEEE Robotics and Automation Letters, 2(2), 688-695. https://doi.org/10.1109/LRA.2017.2650150

Mur-Artal, R., Tardos, J. D., 2017: ORB-SLAM2: An OpenSource SLAM System for Monocular, Stereo, and RGB-D Cameras. IEEE Transactions on Robotics, 33(5), 1255-1262. https://doi.org/10.1109/TRO.2017.2705103

OpenCV: Detection of ArUco Markers., n.d.:

https://docs.opencv.org/master/d5/dae/tutorial_aruco_detection. html (21 January 2021)

Sattler, T., Maddern, W., Toft, C., Torii, A., Hammarstrand, L., Stenborg, E., Safari, D., Okutomi, M., Pollefeys, M., Sivic, J., Kahl, F., Pajdla, T., 2018: Benchmarking 6DOF Outdoor Visual Localization in Changing Conditions. Proceedings of the IEEE Computer Society Conference on Computer Vision and Pattern Recognition, 8601-8610.

https://doi.org/10.1109/CVPR.2018.00897

Sturm, J., Engelhard, N., Endres, F., Burgard, W., Cremers, D., 2012: A benchmark for the evaluation of RGB-D SLAM systems. IEEE International Conference on Intelligent Robots and Systems, September 2015, 573-580.

https://doi.org/10.1109/IROS.2012.6385773

Sumikura, S., Shibuya, M., Sakurada, K., 2019: OpenVSLAM: A Versatile Visual SLAM Framework. Proceedings of the 27th ACM International Conference on Multimedia, 2292--2295. https://doi.org/10.1145/3343031.3350539 